\title{
Chrysin or mannitol for treatment of acute kidney injury: Evidence for pharmacokinetic interaction
}

\author{
Heba M. I. Abdallah ${ }^{1 *}$ (D), Sally A. El Awdan ${ }^{1}$, Salma A. El-Marasy¹, Omar A. Ahmed-Farid², Azza Hassan ${ }^{3}$ \\ ${ }^{1}$ Department of Pharmacology, Medical Research Division, National Research Centre (ID: 60014618), Giza, Egypt. \\ ${ }^{2}$ Department of Physiology, National Organization for Drug Control and Research, Giza, Egypt. \\ ${ }^{3}$ Department of Pathology, Faculty of Veterinary Medicine, Cairo University, Giza, Egypt.
}

\begin{tabular}{l}
\hline ARTICLE INFO \\
\hline Received on: 08/02/2021 \\
Accepted on: 15/07/2021 \\
Available online: 05/12/2021 \\
\hline Key words: \\
Acute kidney injury, \\
ischemia-reperfusion, chrysin, \\
mannitol, pharmacokinetics, \\
rats.
\end{tabular}

\begin{abstract}
Acute kidney injury (AKI) is a heterogeneous disorder that is associated with high percent morbidity and mortality especially in hospitalized patients. Seeking new drugs that have pleiotropic effects on multiple pathologic pathways to treat AKI is a demand. The study aims to investigate and compare the protective effects of chrysin, mannitol, and their combination on an ischemia-reperfusion (I/R) injury rat model. Rats were allocated into 5 groups: sham-operated group, I/R control group, and three groups administered with chrysin $(50 \mathrm{mg} / \mathrm{kg}$, p.o.), mannitol (0.75 ml/100 g bw of 20\% mannitol, i.p.), and chrysin + mannitol, respectively. Biochemical parameters were measured. Histological examination, immunohistochemical analysis of apoptotic markers (caspase-3 and Bax), and pharmacokinetic (PK) study were also performed. Chrysin improved kidney injury indicators, malondialdehyde (MDA), reduced glutathione, inflammatory mediators (prostaglandin E2 and cyclooxygenase-2), and interleukin 1-beta that were deteriorated after I/R injury. It also protected against increased renal damage score and expression of caspase- 3 and Bax. The renoprotective effect of chrysin was comparable to mannitol; however, the combination of both drugs resulted in weak protection against renal injury and showed a decrease in PK parameters $C_{\max }$ and area under the plasma concentrationtime curve as compared to chrysin alone. Both chrysin and mannitol protect against AKI after ischemia-reperfusion via multitarget mechanisms. The combination resulted in a PK interaction.
\end{abstract}

\section{WHAT IS ALREADY KNOWN?}

- Mannitol is the only Food and Drug Administration (FDA)approved treatment for phase oliguric phase of acute kidney injury (AKI).

- However, it has been meeting failure in some clinical settings.

\section{WHAT THIS STUDY ADDS}

- Demonstration of the nephroprotective effect of chrysin in a renal ischemia-reperfusion rat model for the first time.

- The potential mechanisms of mannitol or chrysin nephroprotection are cytoprotective, antioxidant, antiinflammatory, and antiapoptotic activities.

\footnotetext{
"Corresponding Author

Heba Mohammed Ibrahim Abdallah, Department of Pharmacology, Medical Research Division, National Research Centre (ID: 60014618), Giza, Egypt..E-mail: hm.abdullah@nrc.sci.eg
}

\section{CLINICAL SIGNIFICANCE}

- Nephroprotective effect of chrysin was comparable to mannitol. - Combination of chrysin with mannitol resulted in pharmacokinetic interaction which minimized their individual nephroprotective effects and abolished any additive effect of the combination.

\section{INTRODUCTION}

Acute kidney injury (AKI) is a major problematic clinical situation worldwide especially in hospitalized patients as it confers to high mortality rate up to $17 \%$ in ICU cases (Santos and Monteiro, 2015). Ischemic injury has been reported to contribute to about $80 \%-90 \%$ of the etiology of AKI and accounts for more than $50 \%$ of mortality cases as a result of ischemia-reperfusion (I/R) (Bonventre and Yang, 2011). Duration of ischemia affects the severity of I/R injury, and further, during the reperfusion phase, additional damage is caused due to reinstitution of essential blood flow to the ischemic tissue (Kennedy and Erlich, 2008). Renal 
ischemia usually occurs after renal transplantation, shock, sepsis, and renal artery stenosis (Chander and Chopra, 2006). Cellular mechanisms including the high generation rate of reactive oxygen species (ROS), endothelial cell injury, and apoptosis are implicated in the pathogenesis of early phases of renal I/R injury (Bonventre and Weinberg, 2003). Besides, there is accumulating evidence that $\mathrm{I} / \mathrm{R}$ injury results in an inflammatory disease, as manifested by the infiltration of leucocytes, upregulation of chemotactic factors by endothelial cells, and generation of proinflammatory mediators by renal tubular epithelial cells (Ysebaert et al., 2004).

Indeed, AKI is a heterogeneous disorder that is associated with short- and long-term morbidity and mortality in hospitalized patients. Additionally, the AKI insult always precedes its detection and it is therefore difficult to intervene before the disease is established. Early identification and treatment of AKI with drugs that have pleiotropic effects on multiple pathologic pathways are still challenging.

Mannitol is an osmotic diuretic that is commonly used to prevent acute renal failure. It is the only FDA-approved treatment for the oliguric phase of AKI since 1994. Mannitol is freely filtered at the glomerulus and undergoes limited reabsorption by renal tubules. It acts by extracting water from intracellular compartments, expanding the extracellular fluid volume. So, it decreases blood viscosity and inhibits renin release (Karajala et al., 2009). Mannitol is also a free radical scavenger that removes obstructing tubular casts and dilutes the nephrotoxic substances present in the tubular fluid during reperfusion of the kidney. These actions lead to an increase in renal blood flow (Shawkat et al., 2012). Animal models have demonstrated the renoprotective effect of mannitol due to acute tubular necrosis, I/R injury (Özlülerden et al., 2017), contrast-induced nephropathy (Kalogirou et al., 2019), and kidney damage associated with pancreatic I/R (Khy et al., 2010). So, further experimental evidence for protective cellular mechanisms of mannitol against renal injury is crucial to support its clinical use for early AKI prevention.

Chrysin is a natural flavonoid found in many plant extracts including the blue passion flower, honey, and bee propolis. It has several pharmacological effects as antioxidant, anti-inflammatory, antiaging, and anticancer (Ali et al., 2014). Its antiasthmatic activity is attributed to the suppression of inducible nitric oxide synthase and nuclear factor-kB (Wadibhasme et al., 2011), anti-inflammatory activity via blocking histamine release and proinflammatory cytokine expression, and prevention of osteoporosis by activation of estrogen receptor/mitogenactivated protein kinase (Zeng et al., 2013). Chrysin also showed renoprotective activity by suppressing vascular endothelial growth factor-induced angiogenesis (Samarghandian et al., 2017). However, up till now, chrysin was not tested in the experimental model of I/R predisposing to early AKI.

Taking together, to fulfill these gaps, the present study was constructed and aimed to investigate and compare the possible renoprotective effects of chrysin, mannitol, or their combination against renal injuries caused by renal ischemia/reperfusion in rats.

\section{MATERIAL AND METHODS}

\section{Animals}

Male albino Wistar rats weighing 180-200 g were purchased from the Animal House Colony at the National Research
Centre, Dokki, Cairo, Egypt. Animals were left to acclimatize at least 7 days before starting the experiment and were kept at standard housing conditions $\left(24^{\circ} \mathrm{C} \pm 1^{\circ} \mathrm{C}\right.$ and 12 hours light/dark cycle). Rats were supplied with standard laboratory pellets and water ad libitum.

\section{Ethical aspects}

All animal procedures were conducted in compliance with the Ethics Committee of the National Research Centre for Animal Care and Use (approval number: 18/128), which is compatible with Canadian Council on Animal Care.

\section{Drugs and chemicals}

Chrysin, the pure flavonoid compound (purity: $\geq 97 \%$ ), and mannitol (purity: $\geq 98 \%$ ) were purchased from Sigma-Aldrich Chemie GmbH (Taufkirchen Germany). All other reagents used in the experiments were of analytical grade and of the highest purity.

\section{Experimental design}

Forty rats were randomly allocated into 5 groups $(8$ per group): Group I: rats were subjected to laparotomy without occluding or clamping the renal artery and served as the shamoperated group, Group II: rats were subjected to renal I/R (as described below) and served as the I/R control group, and Groups III-V: rats were administered with chrysin $(50 \mathrm{mg} / \mathrm{kg}$, p.o.), mannitol $(0.75 \mathrm{ml} / 100 \mathrm{~g}$ of weight of $20 \%$ mannitol, i.p.), and chrysin + mannitol, respectively, for 21 days before renal I/R. The sham-operated and $\mathrm{I} / \mathrm{R}$ renal groups similarly received the vehicle for 21 days. The selection of the doses of chrysin and mannitol was based on previously published data of Rashid et al. (2016) and Olguín et al. (2009), respectively.

\section{Induction of renal $I / R$}

Rats were anesthetized with chloral hydrate $(300 \mathrm{mg} / \mathrm{kg}$ i.p.) 60 minutes following the last dose administration. A midline abdominal incision was done to expose the left kidney. The left renal artery was occluded with a microvascular clamp for 30 minutes. Ischemia was confirmed by the blanching of the kidney. After 30 minutes, the clamp was removed and renal reperfusion was observed visually. The wound was sutured with silk suture and the rats were allowed to recover (Choucry et al., 2018). The sham-operated group was subjected to the same conditions but without $\mathrm{I} / \mathrm{R}$ induction.

\section{Sample and tissue collection}

24 hours following reperfusion, blood samples were collected from the retro-orbital venous plexus for estimating serum biochemical parameters. The serum was separated by centrifugation at $1,538 \mathrm{~g}$ for 10 minutes. Then, rats were euthanized by decapitation; the left kidney was separated and divided into two longitudinal sections. One section was stored at $-80^{\circ} \mathrm{C}$ for further biochemical analysis and the other section for histopathological examination and immunohistochemical analysis.

\section{Renal homogenate preparation}

The left kidney was homogenized (MPW-120; Medical Instruments) in $20 \%(w / v)$ ice-cold phosphate buffer. Then, the homogenate was centrifuged using a cooling centrifuge $(2 \mathrm{k} 15$; 
Sigma/Laborzentrifugen) at 1,538 $\mathrm{g}$ for 5 minutes and the resulting supernatant was used for determining the renal biochemical parameters.

\section{Assessment of renal function}

Serum creatinine was determined using biodiagnostic kits (Cairo, Egypt) according to the method described by Spencer (1986). Serum lactate dehydrogenase (LDH) was assessed by the method described by Pesce (1984) using Chronolab kits (Spain).

\section{Assessment of oxidative stress biomarkers}

Lipid peroxidation was determined in renal tissue by measuring malondialdehyde (MDA) renal content using the thiobarbituric reactive substance as described by Ruiz-Larrea et al. (1994). Reduced glutathione (GSH) renal content was assessed according to the method of Ellman (1959).

\section{Assessment of inflammatory mediators}

Prostaglandin E2 (PGE2), cyclooxygenase-2 (COX-2), and interleukin 1-beta (IL-1 $\beta$ ) were determined in renal tissue using PGE2, COX-2, and IL-1 $\beta$ enzyme-linked immunosorbent assay kits, respectively, obtained from Glory Science (Del Rio, Texas, USA) following the manufacturer's instructions.

\section{Histopathological examination and assessment of renal tubular damage}

Tissue samples from right and left kidneys from animals subjected to ischemia and other treated animals as well as the normal one were fixed in $10 \%$ neutral formalin for at least 24 hours. The tissues were processed and embedded in paraffin wax. $4 \mu \mathrm{m}$ thick sections were stained with hematoxylin and eosin stain $(\mathrm{H} \& \mathrm{E})$. Ten random microscopic fields $(20 \times)$ per section were examined for assessment of renal tubular damage. The main pathological criteria used for this assessment were tubular necrosis, eosinophilic cast, and tubular dilatation. The extent of renal damage was quantitatively scored according to the method of Muratsubaki et al. (2017), with some modifications, in which score $1=<10 \% ; 2=10 \%-50 \% ; 3=50 \%-<90 \%$; and $4=>90 \%$.

\section{Immunohistochemical investigation}

Determination of activated caspase-3 and Bax expression as apoptotic markers was carried out on paraffinembedded renal tissue as previously described (Hegazy et al., 2016). The sections were dewaxed and incubated in $0.03 \%$ hydrogen peroxide. Then, the sections were incubated with rabbit polyclonal anticaspase-3 (diluted to 1:1,000, Abcam, Ltd., USA) and rabbit polyclonal anti-Bax (1:200, Santa Cruz Biotechnology). Demonstration of peroxidase activity was achieved through staining with diaminobenzidine tetrahydrochloride (DAB, Dako). Counterstaining with Harris hematoxylin was performed. For assessment of the immunohistochemical analysis, two main criteria including the staining intensity and the percentage of positively stained cells were independently evaluated in ten random high power fields $(40 \times)$ according to the method of Eschwege et al. (1998) with some modifications. The staining intensity was estimated on a scale score graded from 0 to 3 in which $0=$ no staining, $1=$ weak staining, $2=$ moderate staining, and $3=$ strong staining. On the other hand, the percentage of the stained area was estimated using a 4-point scale, in which $0=0 \%, 1=<25 \%, 2=$ $25 \%-60 \%$, and $3=>60 \%$. The total immunoreactivity score (IRS) for each tissue section is the sum of the two criteria.

\section{Pharmacokinetic (PK) study}

Eighteen adult male Sprague Dawley rats, weighing 180-200 g each, were brought from the animal house of the National Organization for Drug Control and Research (NODCAR, Giza, Egypt). All animals received humane care in compliance with the guidelines of the animal care and use of the committee of NODCAR. Animals were kept in normal condition and kept for 1 week for adaptation before the experiment. They were fed a standard diet and free access to water. Rats were allocated into 3 groups (each group 6 rats) as follows: Group1 received mannitol $(0.75 \mathrm{ml} / 100 \mathrm{~g}$ of weight of $20 \%$ mannitol, i.p.). Group 2 received chrysin (50 mg/kg, p.o.). Group 3 received mannitol concurrent with chrysin at the same doses. Plasma samples were collected at $0.25,0.5,1,2,4,6,8,12$, and 24 hours after dose.

Chrysin was determined by high-performance liquid chromatography (HPLC) according to the modified method of Bruschi et al. (2003). Chromatographic condition: mobile phase was conducted as gradient system between A (methanol) and B (acetonitrile 97.5: $\mathrm{H} 2 \mathrm{O} 2.5$ ) for 37 minutes ( 0 minutes started with $50 \%$ A-50\% B, 28 minutes $80 \%$ A-20\% B, 30 minutes $100 \%$ A, 35 minutes $100 \% \mathrm{~A}$, and 37 minutes $50 \% \mathrm{~A}$ and $50 \% \mathrm{~B}$ ); the column was RP C18 $(250 \times 4.6 \mathrm{~mm})$; temperature was $30^{\circ} \mathrm{C}$; the wavelength was $310 \mathrm{~nm}$. Mannitol was determined by HPLC according to the modified method of Kiyoshima et al. (2002). The separation of mannitol in plasma was devised via HPLC. The aliquot volume of serum was mixed with chloroform and methanol and phosphate buffer ( $\mathrm{pH}$ 7.0). After centrifugation, an aliquot of the aqueous layer was evaporated to dryness and derivatized with p-nitrobenzoyl chloride at $50^{\circ} \mathrm{C}$ for 1 hour. Chromatographic condition: column used was RP C18 $(250 \times 3 \mathrm{~mm}$ i.d., $5 \mathrm{~mm})$, the temperature was $20^{\circ} \mathrm{C}$, the mobile phase was acetonitrile $75 \%$ and $25 \% \mathrm{H} 2 \mathrm{O}$ containing $0.05 \%$ trifluoroacetic acid, $0.05 \%$ heptafluoro-n-butyric acid, $0.1 \%$ triethylamine, and the flow rate, $1 \mathrm{ml} /$ minute, and the wavelength was $260 \mathrm{~nm}$.

\section{Statistical analysis}

Data analysis was performed blindly by one-way analysis of variance (ANOVA) for biochemical parameters. Tukey's multiple comparison test was used as post hoc to test statistical significance at $p<0.05$. Results are expressed as mean \pm standard error of mean (SEM) $(n=6)$. The immunohistochemical determination of Bax and caspase- 3 was analyzed using one-way ANOVA followed by the Newman-Keuls multiple comparison test as post hoc test.

The PK parameters were obtained by the maximum concentration $\left(C_{\max }\right)$ and the corresponding time $\left(T_{\max }\right)$ and then were directly obtained from the curves. The trapezoidal method was adopted to calculate the area under the plasma concentrationtime curve (AUC), volume of distribution (VD), $t^{1} \frac{1}{2}$, elimination constant $(\mathrm{Ke})$, and clearance $(\mathrm{CL})$. Data were subjected to the general linear methods procedures of the statistical analysis system (SAS) statistical system package (SAS 2003) and represented as mean $\pm \mathrm{SD}, n \pm 6$ 


\section{RESULTS}

\section{Serum biochemical parameters}

Renal $\mathrm{I} / \mathrm{R}$ injury showed a significant rise in serum LDH and creatinine levels by approximately 3.52- and 1.3-fold as compared to sham rats, respectively, as shown in Table 1. Oral pretreatment with chrysin $(50 \mathrm{mg} / \mathrm{kg})$ for 21 consecutive days resulted in a significant reduction of serum LDH and creatinine levels to be $60.08 \%$ and $74.36 \%$ as compared to renal $\mathrm{I} / \mathrm{R}$ rats, respectively. Mannitol pretreatment $(0.75 \mathrm{ml} / 100 \mathrm{~g}$ of weight of $20 \%$ mannitol, i.p.) for 21 consecutive days significantly reduced serum LDH and creatinine levels to be $50.30 \%$ and $64.10 \%$ as compared to renal I/R rats, respectively. Combined oral pretreatment of chrysin and mannitol still showed an elevated LDH level similar to renal I/R rats and significantly reduced elevated serum creatinine level to be $69.23 \%$ as compared to renal $\mathrm{I} / \mathrm{R}$ rats.

\section{Renal oxidative stress biomarkers}

As shown in Table 2, renal I/R rats showed a significant increase in MDA content and a significant reduction in GSH content as compared to sham-operated rats. Chrysin pretreatment significantly reduced the elevated renal MDA content to be $79.87 \%$ and increased renal GSH content to be $129.54 \%$ as compared to renal I/R rats. Intraperitoneal mannitol pretreatment showed a significant reduction in renal MDA content to be $118.30 \%$ and restored renal GSH to be $118.30 \%$ as compared to renal I/R rats. Pretreatment with chrysin and mannitol restored renal MDA and
GSH contents to be 83.96 and $128.76 \%$ when compared to renal $\mathrm{I} / \mathrm{R}$ rats.

\section{Renal inflammatory biomarkers}

As demonstrated in Figure 1a-c, renal I/R rats resulted in a significant increase in renal PGE2, Cox-2, and IL- $1 \beta$ contents by approximately $3.50-, 1.42-$, and 1.20 -fold as compared to sham rats. Oral pretreatment with chrysin showed a decrease in renal PG-E2, Cox-2, and IL-1 $\beta$ contents to be $48.83 \%, 71.19 \%$, and $86.27 \%$ as compared to renal I/R rats, respectively. Administration of mannitol significantly reduced renal PG-E2, Cox-2, and IL-1 $\beta$ contents to be $68.49 \%, 69.86 \%$, and $85.68 \%$ as compared to renal I/R rats. Chrysin and mannitol pretreatment reduced renal Cox-2 and IL- $1 \beta$ contents to be $72.27 \%$ and $83.39 \%$ as compared to renal $\mathrm{I} / \mathrm{R}$ rats, respectively, which were similar to values elicited by each drug alone; however, PG-E2 content was similar to the renal I/R control group.

The original data source for all biochemical parameters is provided in Supplemental Material S1 https://www.japsonline. com/S1.xlsx.

\section{Renal histopathology}

The renal damage score recorded in ischemic kidneys and kidneys of other treated groups is shown in Table 3. Normal kidney histology without significant histopathological lesions was demonstrated in normal rats (Figs. 2a and 1b). Conversely, the kidneys of the I/R group revealed widespread renal damage, with

Table 1. Effect of chrysin or mannitol on serum levels of kidney function in ischemic rats.

\begin{tabular}{ccc}
\hline Group & LDH (U/l) & Serum creatinine $(\mathbf{m g} / \mathbf{d l})$ \\
\hline Sham-operated & $337.94^{*} \pm 0.60$ & $0.9^{*} \pm 0.04$ \\
I/R control & $1181.06^{* *, * * * *} \pm 191.06$ & $1.17^{* *, * * * * *} \pm 0.06$ \\
Chrysin & $756.88^{* *, * *} \pm 88.69$ & $0.87^{*} \pm 0.04$ \\
Mannitol & $685.38^{* * * *} \pm 33.79$ & $0.75^{*} \pm 0.03$ \\
Chrysin + mannitol & $921.48^{* *} \pm 18.70$ & $0.81^{*} \pm 0.02$ \\
\hline
\end{tabular}

Results are expressed as mean \pm SEM $(n=6)$. Statistical analysis was carried out by one-way ANOVA followed by Tukey's multiple comparison test.

"Significant difference from the I/R control group at $p<0.05$.

${ }^{* *}$ Significant difference from the sham-operated group at $p<0.05$.

${ }^{* * *}$ Significant difference from the mannitol group at $p<0.05$.

Table 2. Effect of chrysin and or mannitol on renal MDA and GSH contents in ischemic rats.

\begin{tabular}{ccc}
\hline Group & MDA $(\mathbf{n m o l} / \mathbf{m g}$ tissue $)$ & GSH $(\boldsymbol{\mu m o l} / \mathbf{g}$ tissue $)$ \\
\hline Sham-operated & $194.10^{*} \pm 11.31$ & $9.66^{*} \pm 0.24$ \\
I/R control & $274.68^{* * * * * * *} \pm 13.19$ & $7.65^{* * * * * * *} \pm 0.20$ \\
Chrysin & $219.39^{*} \pm 0.65$ & $9.91^{*} \pm 0.5$ \\
Mannitol & $208.65^{*} \pm 4.68$ & $9.05 \pm 0.43$ \\
Chrysin + mannitol & $230.61^{*} \pm 14.55$ & $9.85^{*} \pm 0.43$ \\
\hline
\end{tabular}

Results are expressed as mean $\pm \operatorname{SEM}(n=6)$. Statistical analysis was carried out by one-way ANOVA followed by Tukey's multiple comparison test.

*Significant difference from the I/R control group at $p<0.05$.

${ }^{* *}$ Significant difference from the sham-operated group at $p<0.05$.

${ }^{* * * *}$ Significant difference from the mannitol group at $p<0.05$. 
a mean renal damage score of $4.00 \pm 0.00$, represented by massive tubular necrosis and apoptosis which are characteristically seen in the renal cortex and outer medulla, associated with periglomerular and peritubular hemorrhage (Fig. 2c). The necrosis of renal tubules is of a coagulative type that is characterized by intensely eosinophilic cytoplasm with complete karyolysis. The necrotic epithelial cells in the renal medulla were desquamated in the tubular lumen along with the presence of hyalinized and granular cast (Fig. 2d). Significant improvement was recorded in chrysin $(50 \mathrm{mg} / \mathrm{kg}$, p.o.) $+\mathrm{I} / \mathrm{R}$, with a mean renal damage score of $3.00 \pm 0.21$. The pathological lesions are characterized by necrosis and apoptosis of individual cells lining proximal convoluted tubules of the renal cortex associated with tubular dilatation (Fig. 2e) and intraluminal cellular and hyaline cast in the distal convoluted tubules of the renal

Table 3. Effect of chrysin and or mannitol on the mean renal damage score in ischemic rats.

\begin{tabular}{cc}
\hline Group & Renal damage score \\
\hline Sham-operated & $1.00^{\mathrm{d}} \pm 0.00$ \\
I/R control & $4.00^{\mathrm{a}} \pm 0.00$ \\
Chrysin & $3.00^{\mathrm{b}} \pm 0.21$ \\
Mannitol & $2.00^{\mathrm{c}} \pm 0.21$ \\
Chrysin + mannitol & $3.33^{\mathrm{b}} \pm 0.16$ \\
\hline
\end{tabular}

Results are expressed as mean $\pm \operatorname{SEM}(n=6)$. Statistical analysis was carried out using one-way ANOVA followed by the Newman-Keuls multiple comparison test. Different symbols are significantly different from each other. medulla (Fig. 2f). Similarly, marked amelioration was recorded in mannitol $+\mathrm{I} / \mathrm{R}$ groups (the mean renal damage score is $2.00 \pm$ 0.21 ), in which the necrotic renal tubules were greatly regressed and the necrosis was restricted to single cells in both renal cortex and medulla (Fig. $2 \mathrm{~g}$ and $\mathrm{h}$ ). However, mild amelioration was observed in the chrysin + mannitol $+\mathrm{I} / \mathrm{R}$ group (the mean renal damage score is $3.40 \pm 0.16$ ), compared to the mannitol $+\mathrm{I} / \mathrm{R}$ group. Intense periglomerular and peritubular hemorrhage with the presence of brown hemosiderin pigment were characteristic lesions in the renal cortex and medulla. The nuclei in most renal tubules showed pyknotic and karyorrhectic (Fig. 2i and j).

\section{Immunohistochemistry}

The total IRS for evaluation of activated caspase- 3 and Bax expression in ischemic kidneys and kidneys of other treated groups is shown in Figures 3 and 4. Strong and diffuse activated caspase- 3 and Bax expression were recorded in the $\mathrm{I} / \mathrm{R}$ group (the mean IRS $=6.00 \pm 0.00$ and $5.70 \pm 0.15$ for caspase- 3 and Bax, respectively) compared to the normal group. The immune reactivity was demonstrated in the glomerular and renal tubular epithelial cells of the renal cortex and outer medulla in addition to the desquamated epithelium in the tubular lumen (Figs. $3 \mathrm{~b}$ and $4 \mathrm{~b}$ ) for activated caspase-3 and Bax, respectively. No activated caspase-3 and Bax expression were demonstrated in the normal kidneys (Figs. $3 \mathrm{a}$ and $4 a$, respectively). Decreased expression of activated caspase- 3 and Bax was recorded in the chrysin $+\mathrm{I} / \mathrm{R}$ group (the mean IRS $=4.70 \pm 0.21$ and $4.80 \pm 0.21$, respectively) which is significantly different from the $\mathrm{I} / \mathrm{R}$ group. The immune reactivity was mainly a)

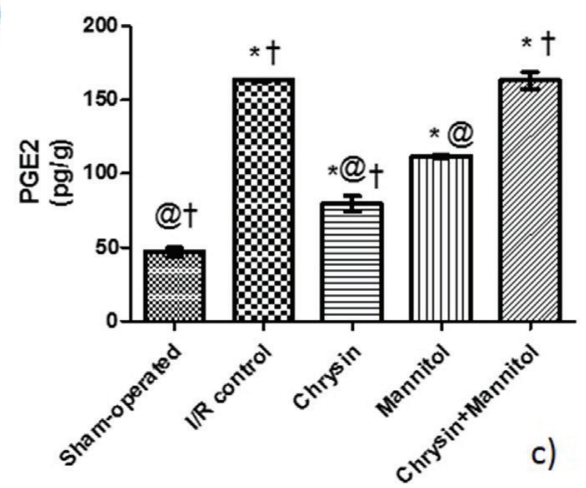

b)

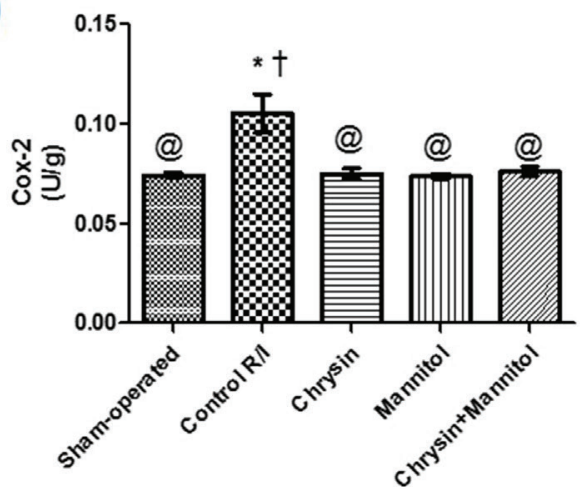

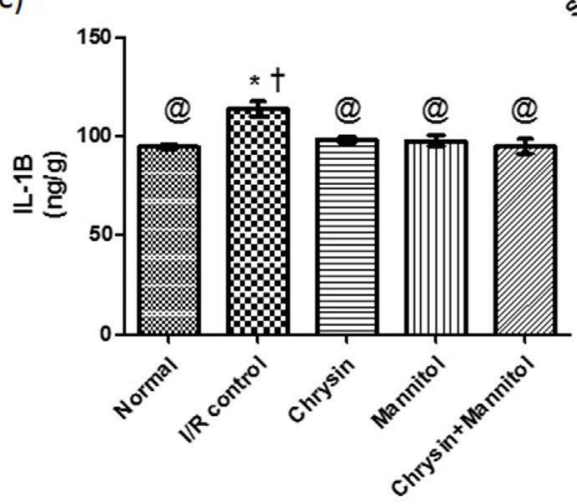

Figure 1. Effect of chrysin and/or mannitol on renal inflammatory markers. (a) PGE2, (b) COX-2, and (c) IL-1 $\beta$. Results are expressed as mean \pm SEM ( $n=$ 6). Statistical analysis was carried out by one-way ANOVA followed by Tukey's multiple comparison test. *Significant difference from the sham-operated group at $p<0.05$. *Significant difference from the $\mathrm{I} / \mathrm{R}$ control group at $p<0.05$. 


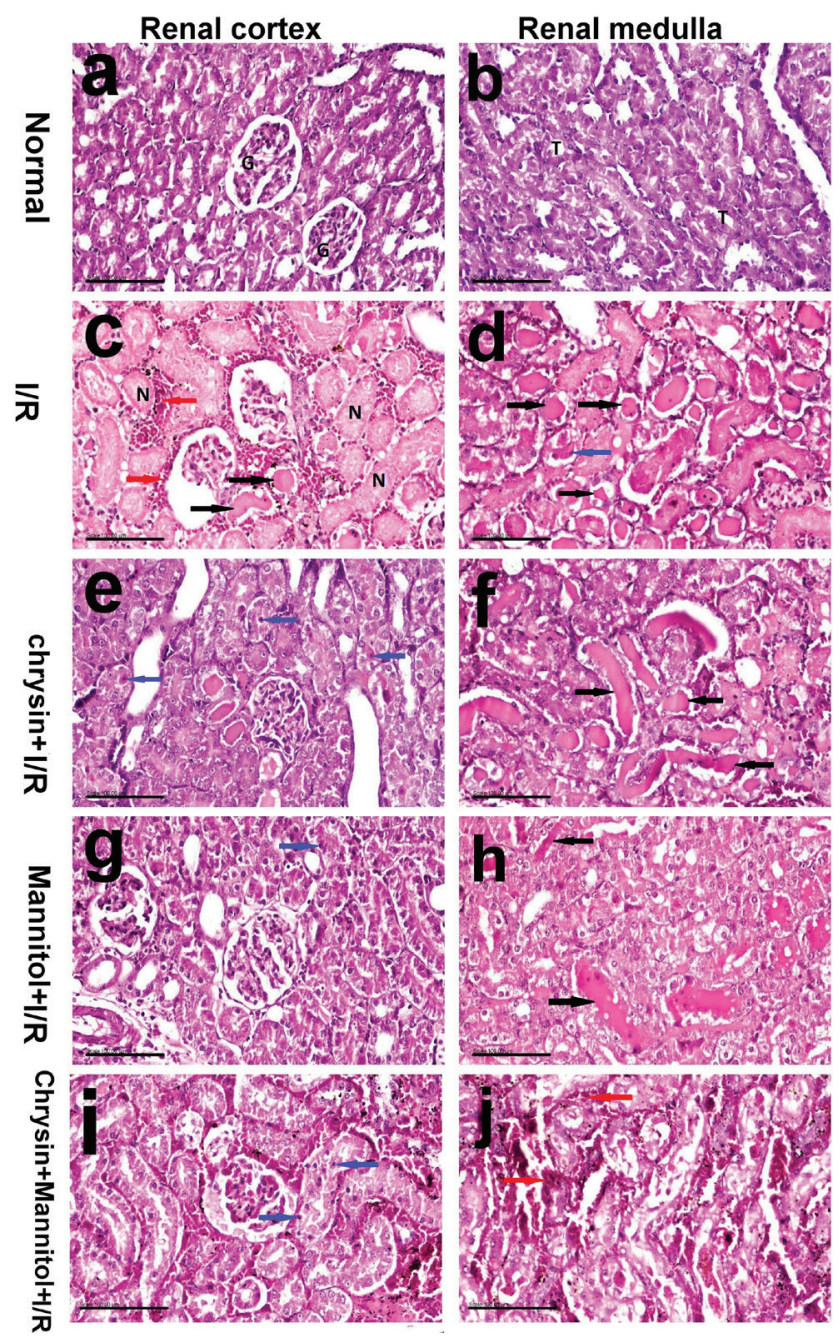

Figure 2. Photomicrograph of renal tissues of normal rats shows normal renal cortex with normal glomeruli (G) (a) and medulla with normal tubules (T) (b); the $\mathrm{I} / \mathrm{R}$ group shows massive tubular necrosis of proximal convoluted tubules $(N)$ of renal cortex associated with periglomerular and peritubular hemorrhage (red arrows) (c) and desquamated necrotic epithelium in the tubular lumen (blue arrow) along with presence of hyalinized and granular cast (black arrows) (d); the chrysin $(50 \mathrm{mg} / \mathrm{kg}$, p.o. $)+\mathrm{I} / \mathrm{R}$ group shows necrosis and apoptosis of individual cells lining proximal convoluted tubules of renal cortex (blue arrows) (e) and intraluminal cellular and hyaline cast in the distal convoluted tubules of renal medulla (black arrows) (f); the mannitol $+\mathrm{I} / \mathrm{R}$ group shows necrosis and apoptosis of single cells lining proximal convoluted tubules (blue arrow) (g) and distal tubules with few intratubular hyaline cast (black arrows) (h); the chrysin + mannitol $+\mathrm{I} / \mathrm{R}$ group shows necrosis of renal tubular epithelium with pyknotic and karyorrhectic nuclei (blue arrows) (i) associated with periglomerular and peritubular hemorrhage (red arrows) (j) (H\&E stain, scale bar $100 \mu \mathrm{m})$.

demonstrated in the renal tubular epithelial cells (Figs. 3c and 4c) for activated caspase-3 and Bax, respectively. Also, a significant decrease of activated caspase- 3 (the mean IRS $=2.80 \pm 0.20$ ) and Bax (the mean IRS $=2.60 \pm 0.20$ ) expression was recorded in the mannitol + I/R group (Figs. 3d and $4 d$ ) for activated caspase-3 and Bax, respectively, which is significantly different from the $\mathrm{I} / \mathrm{R}$ and chrysin $+\mathrm{I} / \mathrm{R}$ groups. Activated caspase- 3 and Bax expression recorded in the chrysin + mannitol $+\mathrm{I} / \mathrm{R}$ group were insignificantly different from the chrysin $+\mathrm{I} / \mathrm{R}$ group (the mean IRS for activated caspase- 3 and Bax are $4.80 \pm 0.20$ and $4.50 \pm 0.16$, respectively)
(Figs. 3e and 4e for activated caspase-3 and Bax, respectively). See Supplemental File S2 https://www.japsonline.com/S2.xlsx for the original source of histopathological and immunohistochemical data.

\section{PK findings}

Obtained data in Table 4 and Figure 5 showed PK interactions of the combination. Administration of chrysin concurrently with mannitol did not affect mannitol PKs except for the little effect on AUC. On the other hand, the combined treatment showed a significant $(p<0.05)$ decrease in the PK parameters $\left(C_{\max }, \mathrm{AUC}, t^{1} / 2\right)$ of chrysin without any changes in $T_{\max }$. There was a significant increase in Ke and CL of chrysin upon the combination with mannitol. The data source is shown in the Supplemental File S3 https://www.japsonline.com/S3.xls.

\section{DISCUSSION}

The current study demonstrates, for the first time, the nephroprotective effect of chrysin on a renal I/R rat model. The study also highlights the potential mechanisms of this nephroprotection such as cytoprotective, antioxidant, antiinflammatory, and antiapoptotic activities. This is also the first time to implicate the anti-inflammatory effect of mannitol in mediating its renoprotective effect. Chrysin, mannitol, or their combination successfully improved biochemical, histopathological, and immunohistochemical events associated with $\mathrm{I} / \mathrm{R}$ induced renal injury.

Renal I/R injury is a valid animal model for studying AKI. It induces a range of histopathological consequences that closely resembles the variable nephron-pathologies seen in patients (Salahudeen et al., 2004). In the present study, ischemic injury to the kidney by 30 minutes occlusion of left renal artery followed by reperfusion for 24 hours induced functional impairment to the kidney represented by increased serum levels of creatinine and tissue injury as indicated by increased serum LDH activity. Creatinine is a product of muscle breakdown that is eliminated by the kidney. Together with blood urea nitrogen, serum creatinine is used as a specific marker for the assessment of renal function (Beier et al., 2011). Despite not being usually sensitive, it is used as a surrogate marker for evaluation of glomerular filtration rate especially in case of difficulty in measuring creatinine CL (AbouHany et al., 2018). Although LDH is a nonspecific marker for cell injury and necrosis, its elevations after renal I/R could reflect cell death and organ tissue damage accurately (Tugtepe et al., 2007). Renal injury severity is proportional to the serum LDH level (Zager et al., 2013).

Measurement of current serum creatinine concentration and LDH activity shows that chrysin, mannitol, and their combination could preserve kidney function by decreasing renal damage after I/R. However, the combination did not elicit a superior effect to each drug alone. The reduction of serum LDH indicates the cytoprotective effect of these investigated drugs and their capability to decrease systemic inflammation as previously mentioned (Yosri et al., 2017). Similarly, alleviation of renal ischemic injury was associated with lower serum creatinine as in other reports (Zhang et al., 2018). In parallel, histopathological abnormalities have been observed in the current investigation after $\mathrm{I} / \mathrm{R}$ injury such as massive tubular necrosis and apoptosis seen in the renal cortex and outer medulla, associated with periglomerular 


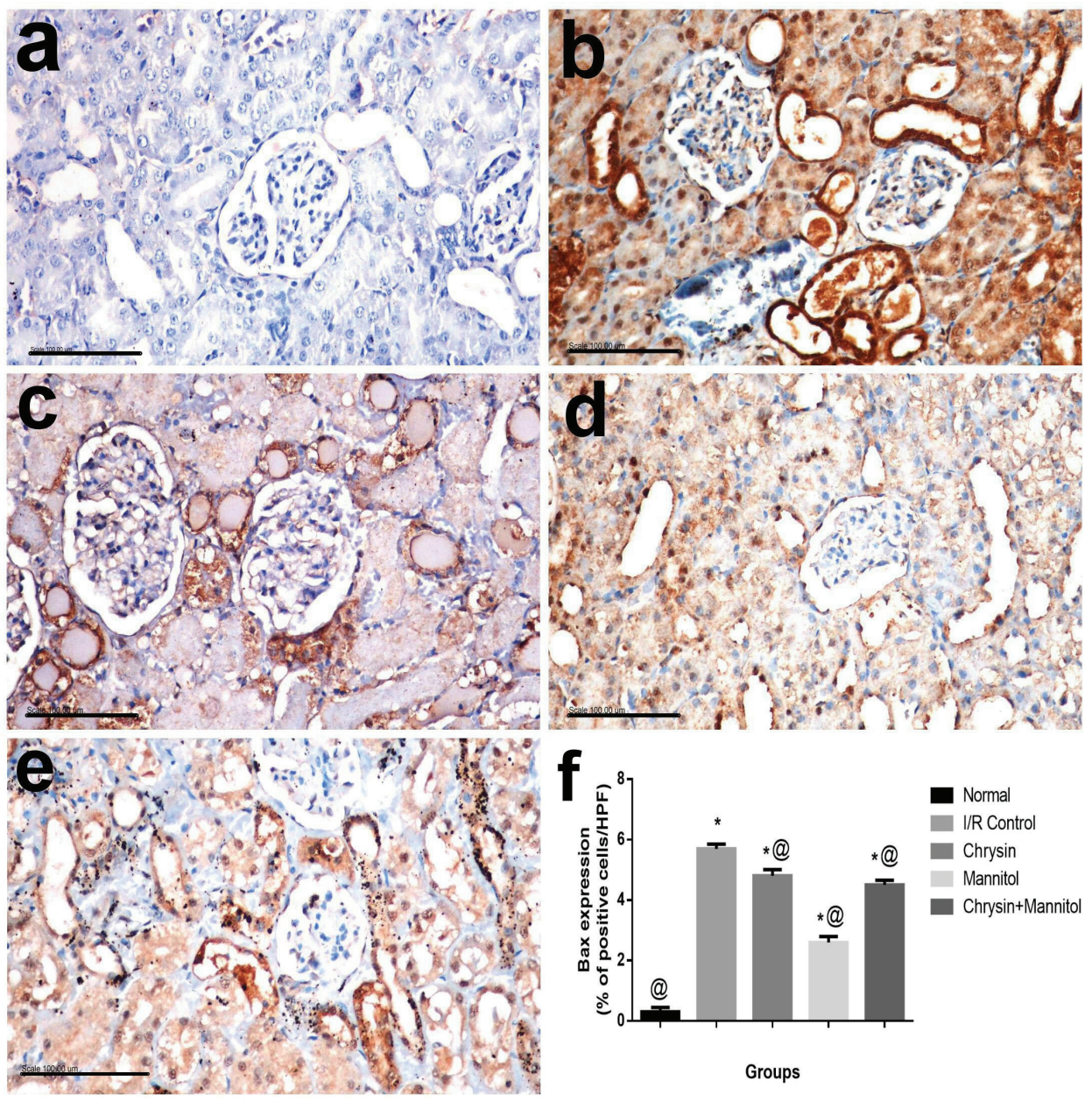

Figure 3. Bax expression in the renal tissue of ischemic and treated groups. Renal tissues of (a) normal rats show no immune staining, (b) the I/R group shows strong Bax expression in the glomerular and renal tubular epithelial cells as well as the desquamated epithelium in the tubular lumen, (c) the chrysin (50 mg/kg, p.o.) + I/R group shows decreased Bax expression, (d) the mannitol $+\mathrm{I} / \mathrm{R}$ group shows a significant decrease of Bax expression in the renal tubular epithelial cells, and (e) the chrysin + mannitol $+\mathrm{I} / \mathrm{R}$ group shows increased Bax expression in the renal tubular epithelial cells (Bax immunohistochemical staining, scale bar $100 \mu \mathrm{m}$ ).

and peritubular hemorrhage associated with high renal damage score. This agrees with previous studies (El-Sisi et al., 2016). These results are consistent with previous studies (Furuichi et al., 2008; Kim et al., 2015), which demonstrated the lesions in the cortex and outer medulla. The massive necrosis and apoptosis seen in the epithelium of the proximal tubules are because of their high metabolic demands and the first line of exposure.

These histopathological findings were also improved by preadministration of chrysin or mannitol confirming their nephroprotection. However, mild amelioration was observed after the combination of chrysin with mannitol regarding renal damage score and tubular necrosis and apoptosis.

Oxidative stress is known to have a central role in the enhancement of I/R renal injury causing exhausting natural antioxidants like GSH and membrane lipid peroxidation as well as DNA oxidative damage and protein oxidation (Aksu et al., 2015). Administration of mannitol, chrysin, or their combination decreased lipid peroxidation as manifested by decreased MDA and increased GSH content in the kidneys of ischemic rats. This implies the protective role that these drugs play against I/R injury through inhibition of free radicals and oxidant formation. In agreement, chrysin was proved to be a powerful antioxidative natural agent that enhanced body natural antioxidant defense and decreased oxidative damage and lipid peroxidation in renal dysfunction due to diabetes mellitus or renal toxicity induced by 5 -fluorouracil (Ahad et al., 2014; Rashid et al., 2016). However, the antioxidant effect of chrysin was not studied in the renal $\mathrm{I} / \mathrm{R}$ ischemic model. Similarly, mannitol is a free radical scavenger that is known to reduce oxidant-derived injury to the kidney (Haraldsson et al., 1995; Weinbroum et al., 1999). Its antioxidant capacity made it a suitable protectant against not only renal ischemia damage but also aortic and lower limb muscular ischemic injury. It was shown that mannitol decreased xanthine oxidase activity and increased GSH level and thus attenuated kidney damage after pancreatic I/R (Goksin et al., 2011; Khy et al., 2010; Weinbroum et al., 2000).

Inflammation is commonly triggered by oxidative stress in the course of ischemic injury as ROS signals the generation of inflammatory cytokines and chemokines. This requisition of inflammatory cells and chemotaxis at the stressed site potentiates more cellular damage (Abou-Hany et al., 2018). Additionally, damaged cells concentrate intracellular calcium up to 10000-fold more than intact cells. This enhances consequent inflammatory 


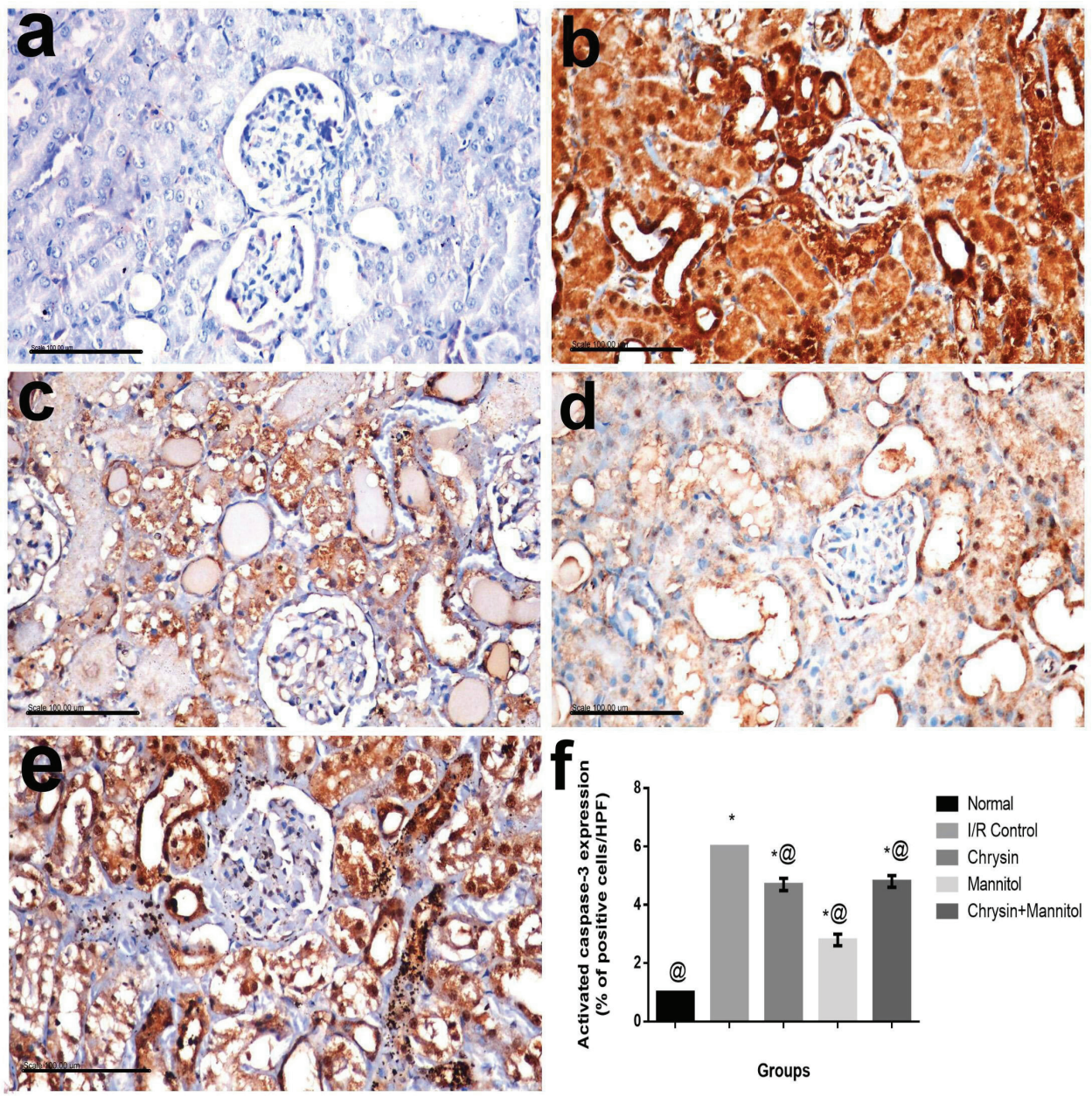

Figure 4. Activated caspase-3 expression in the renal tissue of ischemic and treated groups. Renal tissues of (a) normal rats show no immune staining, (b) the I/R group shows strong and diffuse immunostaining for activated caspase-3 in the glomerular and renal tubular epithelial cells, (c) the chrysin (50 mg/kg, p.o.) $+\mathrm{I} / \mathrm{R}$ group shows a decreased expression of activated caspase- 3 , (d) the mannitol + I/R group shows a significant decrease of activated caspase- 3 expression, and (e) the chrysin + mannitol $+\mathrm{I} / \mathrm{R}$ group shows an intense expression of activated caspase-3 (activated caspase-3 immunohistochemical staining, scale bar $100 \mu \mathrm{m}$ ).

Table 4. PK parameters of mannitol, chrysin, and their combination after simulation route and dose of administration in rats.

\begin{tabular}{ccccc}
\hline \multirow{2}{*}{ Parameters } & \multicolumn{4}{c}{ Groups } \\
\cline { 2 - 5 } & Mannitol & $\begin{array}{c}\text { Mannitol concurrent with } \\
\text { chrysin }\end{array}$ & Chrysin & $\begin{array}{c}\text { Chrysin concurrent with } \\
\text { mannitol }\end{array}$ \\
\hline$T_{\max }$ & 0.25 & 0.25 & 2 & 2 \\
$C_{\max }$ & $47.3 \pm 5.173$ & $47.3 \pm 5.593$ & $7.3 \pm 0.62$ & $5.7 \pm 0.485^{*}$ \\
VD & $3,174 \pm 292$ & $3,171 \pm 277$ & $1,364 \pm 140$ & $1,757 \pm 162^{*}$ \\
$\mathrm{AUC}$ & $145.58 \pm 15.53$ & $129.6 \pm 12.399^{*}$ & $142.03 \pm 12.195$ & $44.94 \pm 4.691^{*}$ \\
$t^{1 / 2}$ & $1 \pm 0.085$ & $0.928 \pm 0.086$ & $3.915 \pm 0.33$ & $2.214 \pm 0.216^{*}$ \\
$\mathrm{Ke}$ & $0.693 \pm 0.062$ & $0.747 \pm 0.066$ & $0.177 \pm 0.021$ & $0.313 \pm 0.033^{*}$ \\
$\mathrm{CL}$ & $0.218 \pm 0.023$ & $0.236 \pm 0.022$ & $0.13 \pm 0.015$ & $0.178 \pm 0.015^{*}$ \\
\hline
\end{tabular}

Values represent mean $\pm \mathrm{SD}(n=6)$.

*Values within column with the same treatment and its combination with unlike superscripts differ significantly $(p<0.05)$, according to paired $t$-test. 


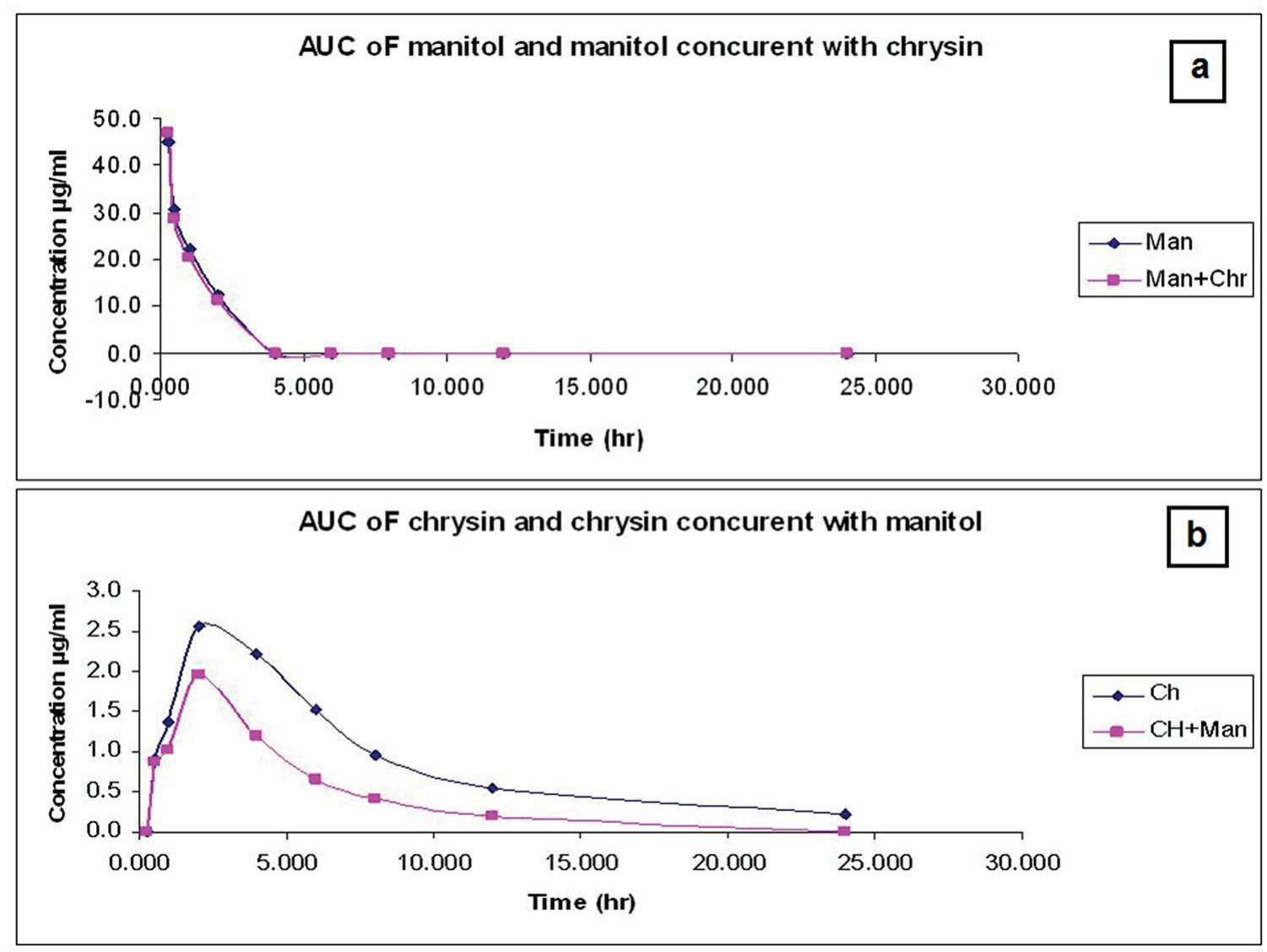

Figure 5. (a) The time concentration relationship of chrysin $(50 \mathrm{mg} / \mathrm{kg}$ b.wt, p.o.) or chrysin concurrent with mannitol $(0.75 \mathrm{ml} / 100 \mathrm{~g} \mathrm{bw}$ of $20 \% \mathrm{mannitol})$ in male albino rats (mean $\pm \mathrm{SD}, n=6$ ). (b) The time concentration relationship of mannitol ( $0.75 \mathrm{ml} / 100 \mathrm{~g}$ bw of $20 \%$ mannitol) or mannitol concurrent with chrysin (50 mg/ $\mathrm{kg}$ b.wt) after oral administration in male albino rats (mean $\pm \mathrm{SD}, n=6$ ).

reactions and immunomodulatory pathways (Zhang et al., 2014). $\mathrm{I} / \mathrm{R}$ injury induces COX-2 production which in turn stimulates the production of inflammatory prostaglandins which boost further kidney injury (Chatterjee et al., 2003). Currently administrated drugs, chrysin, mannitol, and their combination could decrease renal IL- $1 \beta$ and COX-2 content. Also, the administered drugs, when given alone, effectively decreased PG-E2 content. This shows that chrysin and mannitol performed their renoprotective effect through the regression of inflammation and its damaging consequences in the ischemic renal tissue. This concurs with other recent studies which confirmed the strong relationship between regression of renal ischemic injury, reduction of inflammatory mediators level, and principally decreased oxidative damage (Ali et al., 2018; El-Sisi et al., 2016). In parallel, chrysin was reported to improve diabetic nephropathy via reduction of inflammation as shown by decreased IL- $1 \beta$ and oxidative stress (Khan et al., 2011). Chrysin also was shown to decrease COX-2 overexpression and PG-E2 production, and thus, it was assumed to be a good anticancer candidate due to its anti-inflammatory activities in renal precarcinogenesis (Rehman et al., 2013). However, the studies investigating the anti-inflammatory effect of mannitol are rare. In one study, mannitol inhibited COX-2 expression induced by Helicobacter pylori and it was suggested that mannitol, through minimizing oxygen radicals, could attenuate the inflammatory mediators in a gastric epithelial cell line (Kim et al., 2002). In another rat model of arthritis, the topical application of mannitol suppressed joint inflammation (Cavone et al., 2012). As an osmotherapeutic drug, mannitol was chosen in Schreibman et al. (2018) aiming to minimize brain injury due to intracranial hemorrhage. Mannitol showed a powerful effect on microglial activation which is the main determinant of the neuroinflammatory response. Yet, it has been recommended by the authors that the anti-inflammatory mannitol effect was still warranted to be explored.

Apoptosis plays an important role in the pathogenesis of $\mathrm{I} / \mathrm{R}$ injury. Apoptotic stimuli induce cytochrome $\mathrm{c}$ release from mitochondria generating a series of reactions that activate caspases and promote subsequent cell death. Caspase- 3 is cleaved, activated, and then closely coupled to upstream proapoptotic signals responsible for cell apoptosis (Hasenour et al., 2013). Bcl2 family of proteins modulates apoptotic pathways. It contains Bcl-2 and Bcl-XL that function as antiapoptotic factors and others like Bax, Bad, or Bcl-Xs that promote apoptosis. Treatments that downregulated the expression of Bax could inhibit apoptotic cell death (Zhang et al., 2018). Induction of renal ischemia in the current study caused apoptotic cell death seen in the histopathological examination and immunohistochemical evaluation of caspase- 3 and Bax which showed increased activity of these two apoptotic markers. In previous reports, chrysin showed an antiapoptotic effect by attenuating the renal expression of Bax and downregulated 
caspase-3 activation (Rashid et al., 2014). Similarly, mannitol showed an inhibitory effect on apoptotic markers. In parallel, previous reports have shown the antiapoptotic effects of mannitol in experimental animals. Kurt et al. (2016) demonstrated that mannitol reduced ROS and apoptosis and it was deduced that mannitol could protect against $\mathrm{I} / \mathrm{R}$ induced in rats by testicular torsion. However, the combination of chrysin with mannitol did not inhibit apoptosis in renal cells. This unexpected nonsynergistic effect of chrysin and mannitol combination in the current study was explained on a PK basis.

According to the screening of PK of mannitol and chrysin, a decline in physiological response was observed in the ischemic renal model. This alteration was persistent upon a combination of mannitol with chrysin. This is due to the osmolarity effect of mannitol which influenced chrysin PK behavior and increased kidney excretion. Coadministration of mannitol resulted in decreased $C_{\text {max }}, \mathrm{AUC}, t^{1 / 2}$. These data are in parallel with Olguín et al. (2009) who reported the effect of mannitol on the PK of amikacin in Wistar rats and concluded that alteration of the PK parameters was observed between the groups. It was concluded that the deterioration of renal function must be considered when mannitol and amikacin are prescribed simultaneously due to altered PK parameters which leads to unexpected pharmacological parameters of both.

The current interaction manifested during the effect of the combination of mannitol and chrysin may be due to the acceleration of hyponatremia exerted by mannitol. This action might affect the aquaporin activity of chrysin for major intrinsic proteins in the membrane of biological cells; hence, this function may be disrupted after mannitol induced hyponatremic effects and thereby deteriorated the protective activity of chrysin (Fiorentini et al., 2015).

On the other hand, in the present study, little effect was observed by chrysin on the PKs of mannitol confined in a little decrease in AUC. Chrysin is known for its low oral bioavailability, because it is subjected to extensive metabolism, and metabolites are eliminated back into the intestine for being hydrolyzed and undergo fecal elimination (Walle et al., 2001). So, there were attempts to enhance its bioavailability via using nanoformulations (Sulaiman et al., 2018). The little interaction of chrysin with mannitol may have resulted from the rapid metabolism of chrysin as reported previously for the neglected effect of chrysin on caffeine PKs (Noh et al., 2016).

\section{CONCLUSION}

Pretreatment with chrysin, mannitol, or their combination could protect against I/R renal injury (an early predisposing factor to AKI) via cytoprotective, anti-inflammatory, antioxidative, and antiapoptotic activities. However, unexpectedly, the combination of chrysin and mannitol did not induce an additive effect, and this could be illustrated on the basis of PK interaction between both drugs. Mannitol decreased pharmacokinetic parameters of chrysin including bioavailability (AUC), maximum absorption $\left(C_{\max }\right)$, half-life $\left(t^{1} / 2\right)$. On the other hand, mannitol increased elimination, and clearance (Ke and CL) of chrysin.

\section{CONSENT TO PARTICIPATE}

Not applicable.

\section{CONSENT FOR PUBLICATION}

Not applicable.

\section{AVAILABILITY OF DATA AND MATERIAL}

Available.

\section{CODE AVAILABILITY}

Not applicable.

\section{AUTHORS' CONTRIBUTIONS}

The authors declare that all data were generated inhouse and that no paper mill was used. Sally A. El Awdan: conceptualization, methodology, data curation, and visualization. Heba M. I. Abdallah: conceptualization, methodology, data curation, writing - review, editing preparation, and correspondence. Salma A. El-Marasy: conceptualization, methodology, software, writing - review, visualization, and investigation. Omar A. H. A. Farid: software, PK methodology, investigation, and writing. Azza Hassan: pathological and immunohistochemical methodology, investigation, and writing.

\section{FUNDING}

There is no funding to report.

\section{CONFLICTS OF INTEREST}

The authors report no financial or any other conflicts of interest in this work.

\section{ETHICS APPROVAL}

Animal experimentation was approved by the Ethics Committee of the National Research Centre for Animal Care and Use (approval number: 18/128).

\section{PUBLISHER'S NOTE}

This journal remains neutral with regard to jurisdictional claims in published institutional affiliation.

\section{REFERENCES}

Abou-Hany HO, Atef H, Said E, Elkashef HA, Salem HA. Crocin reverses unilateral renal ischemia reperfusion injury-induced augmentation of oxidative stress and toll like receptor-4 activity. Environ Toxicol Pharmacol, 2018; 59:182-9.

Ahad A, Ganai AA, Mujeeb M, Siddiqui WA. Chrysin, an antiinflammatory molecule, abrogates renal dysfunction in type 2 diabetic rats. Toxicol Appl Pharmacol, 2014; 279(1):1-7.

Aksu U, Ergin B, Bezemer R, Kandil A, Milstein DM, DemirciTansel C. Scavenging reactive oxygen species using tempol in the acute phase of renal ischemia/reperfusion and its effects on kidney oxygenation and nitric oxide levels. Intensive Care Med Exp, 2015; 3(1):21.

Ali N, Rashid S, Nafees S, Hasan SK, Sultana S. Beneficial effects of chrysin against methotrexate-induced hepatotoxicity via attenuation of oxidative stress and apoptosis. Mol Cell Biochem, 2014; 385(1-2):215-23; doi:10.1007/s11010-013-1830-4

Ali RM, Al-Shorbagy MY, Helmy MW, El-Abhar HS. Role of Wnt4/beta-catenin, Ang II/TGFbeta, ACE2, NF-kappaB, and IL-18 in attenuating renal ischemia/reperfusion-induced injury in rats treated with Vit D and pioglitazone. Eur J Pharmacol, 2018; 831:68-76.

Beier K, Eppanapally S, Bazick HS, Chang D, Mahadevappa K, Gibbons FK. Elevation of blood urea nitrogen is predictive of long-term mortality in critically ill patients independent of "normal" creatinine. Crit Care Med, 2011; 39(2):305-13. 
Bonventre JV, Weinberg JM. Recent advances in the pathophysiology of ischemic acute renal failure. J Am Soc Nephrol, 2003; 14(8):2199-210.

Bonventre JV, Yang L. Cellular pathophysiology of ischemic acute kidney injury. J Clin Invest, 2011; 121(11):4210-21.

Bruschi ML, Franco SL, Gremia o MPD. Application of an HPLC method for analysis of propolis extract. J Liq Chromatogr Relat Technol, 2003; 26(14):2399-409.

Cavone LA, Calosi LB, Cinci LB, Moroni FA, Chiarugi AA. Topical mannitol reduces inflammatory edema in a rat model of arthritis. Pharmacology, 2012; 89:18-21.

Chander V, Chopra K. Protective effect of nitric oxide pathway in resveratrol renal ischemia-reperfusion injury in rats. Arch Med Res, 2006; 37(1):19-26; doi:10.1016/j.arcmed.2005.05.018

Chatterjee PK, Patel NS, Kvale EO, Brown PA, Stewart KN, Britti D. The tyrosine kinase inhibitor tyrphostin AG126 reduces renal ischemia/reperfusion injury in the rat. Kidney Int, 2003; 64(5):1605-19.

Choucry MA, Khalil MNA, El Awdan SA. Protective action of crateva nurvala Buch. Ham extracts against renal ischaemia reperfusion injury in rats via anti-oxidant and anti-inflammatory activities. J Ethnopharmacol, 2018; 214:47-57.

Ellman GL. Tissue sulfhydryl groups. Arch Biochem Biophys, 1959; 82(1):70-7.

El-Sisi AE, Sokar SS, Abu-Risha SE, Ibrahim HA. Combination of tadalafil and diltiazem attenuates renal ischemia reperfusion-induced acute renal failure in rats. Biomed Pharmacother, 2016; 84:861-9.

Eschwege P, Paradis V, Conti M, Loric S, Dumas F, Berteau P. Bcl-2 and Bax expression on rat ischemic kidney. Transplant Proc, 1998; 30(6):2861-2.

Fiorentini D, Zambonin L, Sega FVD, Hrelia S. Polyphenols as modulators of aquaporin family in health and disease. Oxid Med Cell Longev, 2015; 2015:196914.

Furuichi K, Gao JL, Horuk R, Wada T, Kaneko S, Murphy PM. Chemokine receptor CCR1 regulates inflammatory cell infiltration after renal ischemia-reperfusion injury. J Immunol, 2008; 181(12):8670-6; doi:10.4049/jimmunol.181.12.8670

Goksin I, Adali F, Enli Y, Akbulut M, Teke Z, Sackan G. The effect of phlebotomy and mannitol on acute renal injury induced by ischemia/ reperfusion of lower limbs in rats. Ann Vasc Surg, 2011; 25(8):1118-28.

Haraldsson G, Sorensen V, Nilsson U, Pettersson S, Rashid M, Schersten T. Effect of pre-treatment with desferrioxamine and mannitol on radical production and kidney function after ischaemia-reperfusion. A study on rabbit kidneys. Acta Physiol Scand, 1995; 154(4):461-8.

Hasenour CM, Berglund ED, Wasserman DH. Emerging role of AMP-activated protein kinase in endocrine control of metabolism in the liver. Mol Cell Endocrinol, 2013; 366(2):152-62.

Hegazy R, Salama A, Mansour D, Hassan A. Renoprotective effect of lactoferrin against chromium-induced acute kidney injury in rats: involvement of IL-18 and IGF-1 inhibition. PLoS One, 2016; 11(3): 0151486.

Kalogirou TE, Meditskou S, Davidopoulou S, Savvas I, Pitoulias $\mathrm{AG}$, Pitoulias GA. Investigating the possible protective role of direct intraarterial administration of mannitol and NAcetylcysteine and Per Os administration of simvastatin against contrast-induced nephropathy: an experimental study in a rabbit model. Cardiovasc Intervent Radiol, 2019; 42(12):1777-85; doi:10.1007/s00270-019-02304-8

Karajala V, Mansour W, Kellum JA. Diuretics in acute kidney injury. Minerva Anestesiol, 2009; 75(5):251-7.

Kennedy SE, Erlich JH. Murine renal ischaemia-reperfusion injury. Nephrology (Carlton), 2008; 13(5):390-6.

Khan MS, Halagowder D, Devaraj SN. Methylated chrysin, a dimethoxy flavone, partially suppresses the development of liver preneoplastic lesions induced by N-nitrosodiethylamine in rats. Food Chem Toxicol, 2011; 49(1):173-8.

Khy W, Namnesnikov M, Fedorov D, Abu-Gazala S, Weinbroum AA. Mannitol attenuates kidney damage induced by xanthine oxidaseassociated pancreas ischemia-reperfusion. J Surg Res, 2010; 160(1):163-8; doi:10.1016/j.jss.2008.10.016

Kim H, Seo JY, Kim KH. Effect of mannitol on helicobacter pylori-induced cyclooxygenase-2 expression in gastric epithelial AGS cells Pharmacology, 2002; 66:182-9; doi:10.1159/000065532

Kim MG, Kim SC, Ko YS, Lee HY, Jo SK, Cho W. The role of M2 macrophages in the progression of chronic kidney disease following acute kidney injury. PLoS One, 2015; 10(12):e0143961; doi:10.1371/ journal.pone.0143961

Kiyoshima A, Kudo K, Nishida N, Ikeda N. HPLC simultaneous determination of glycerol and mannitol in human tissues for forensic analysis. Forensic Sci Int, 2002; 125:127-33.

Kurt O, Yazici CM, Erboga M, Turan C, Bozdemir Y, Akbas A. Mannitol has a protective effect on testicular torsion: an experimental rat mode. J Pediatr Urol, 2016; 12(3):167.e1-8; doi:10.1016/j. jpurol.2016.01.004

Muratsubaki S, Kuno A, Tanno M, Miki T, Yano T, Sugawara H, Shibata S, Abe K, Ishikawa S, Ohno K, Kimura Y, Tatekoshi Y, Nakata K, Ohwada W, Mizuno M, Miura T. Suppressed autophagic response underlies augmentation of renal ischemia/reperfusion injury by type 2 diabetes. Sci Rep, 2017; 7(1):5311; doi:10.1038/s41598-017-05667-5

Noh K, Oh do G, Nepal MR, Jeong KS, Choi Y, Kang MJ, Kang W, Jeong HG, Jeong TC. Pharmacokinetic interaction of chrysin with caffeine in rats. Biomol Ther (Seoul), 2016; 24(4):446-52; doi:10.4062/ biomolther.2015.197

Olguín HJ, Portugal MC, Pérez JF, Vieyra AC, Pérez CF, Rodríguez AA. Effect of mannitol on the pharmacokinetics of amikacin in wistar rats. Braz Arch Biol Technol, 2009; 52:835-9.

Özlülerden Y, Toktaş C, Aybek H, Küçükatay V, Şen Türk N, Zumrutbas AE. The renoprotective effects of mannitol and udenafil in renal ischemia-reperfusion injury model. Investig Clin Urol, 2017; 58(4):289-95; doi:10.4111/icu.2017.58.4.289.

Pesce AJ. Lactate dehydrogenase. In: Kaplan LA (ed.). Clinical chemistry: theory, analysis, and correlation. 1st edition, Mosby, St. Louis, MO, pp xxvi, 1476, 1984.

Rashid S, Ali N, Nafees S, Hasan SK, Sultana S. Mitigation of 5-fluorouracil induced renal toxicity by chrysin via targeting oxidative stress and apoptosis in wistar rats. Food Chem Toxicol, 2014; 66:185-93.

Rashid S, Nafees S, Vafa A, Afzal SM, Ali N, Rehman MU, Sultana S. Inhibition of precancerous lesions development in kidneys by chrysin via regulating hyperproliferation, inflammation and apoptosis at pre clinical stage. Arch Biochem Biophys, 2016; 606:1-9; doi:10.1016/j. abb.2016.07.004

Rehman MU, Tahir M, Khan AQ, Khan R, Lateef A, Oday $\mathrm{OH}$. Chrysin suppresses renal carcinogenesis via amelioration of hyperproliferation, oxidative stress and inflammation: plausible role of NFkappaB. Toxicol Lett, 2013; 216(2-3):146-58.

Ruiz-Larrea MB, Leal AM, Liza M, Lacort M, de Groot H. Antioxidant effects of estradiol and 2-hydroxyestradiol on iron-induced lipid peroxidation of rat liver microsomes. Steroids, 1994; 59(6):383-8.

Salahudeen AK, Haider N, May W. Cold ischemia and the reduced long-term survival of cadaveric renal allografts. Kidney Int, 2004; 65:713-8

Samarghandian S, Farkhondeh T, Azimi-Nezhad M. Protective effects of chrysin against drugs and toxic agents. Dose Response, 2017; 15(2):1559325817711782; doi:10.1177/1559325817711782

Santos PR, Monteiro DL. Acute kidney injury in an intensive care unit of a general hospital with emergency room specializing in trauma: an observational prospective study. BMC Nephrol, 2015; 16:30.

Schreibman DL, Hong CM, Keledjian K, Ivanova S, Tsymbalyuk $\mathrm{S}$, Gerzanich. Mannitol and hypertonic saline reduce swelling and modulate inflammatory markers in a rat model of intracerebral hemorrhage. Neurocrit Care, 2018; 29:253-63.

Shawkat H, Westwood MM, Mortimer A. Mannitol: a review of its clinical uses. Contin Educ Anaesth Crit Care Pain, 2012; 12:82-5. 
Spencer K. Analytical reviews in clinical biochemistry: the estimation of creatinine. Ann Clin Biochem, 1986; 23(Pt 1):1-25; doi:10.1177/000456328602300101

Sulaiman GM, Jabir MS, Hameed AH. Nanoscale modification of chrysin for improved of therapeutic efficiency and cytotoxicity. Artif Cells Nanomed Biotechnol, 2018; 46(Sup1):708-20; doi:10.1080/216914 01.2018 .1434661

Tugtepe H, Sener G, Biyikli, NK. The protective effect of oxytocin on renal ischemia/reperfusion injury in rats. Regul Pept, 2007; 140:101-8.

Wadibhasme PG, Ghaisas MM, Thakurdesai PA. Antiasthmatic potential of chrysin on ovalbumin-induced bronchoalveolar hyperresponsiveness in rats. Pharm Biol, 2011; 49(5):508-15; doi:10.310 9/13880209.2010.521754

Walle T, Otake Y, Brubaker JA, Walle UK, Halushka PV. Disposition and metabolism of the flavonoid chrysin in normal volunteers. Br J Clin Pharmacol, 2001; 51(2):143-6; doi:10.1111/j.13652125.2001.01317.x

Weinbroum AA, Hochhauser E, Rudick V, Kluger Y, Karchevsky E, Graf E. Multiple organ dysfunction after remote circulatory arrest: common pathway of radical oxygen species? J Trauma, 1999; 47(4):691-8.

Weinbroum AA, Kluger Y, Rudick V. Impairment of aortal tone by no flow-reflow conditions and its partial amelioration by mannitol. Ann Thorac Surg, 2000; 69(5):1439-44.

Yosri H, Elkashef WF, Said E, Gameil NM. Crocin modulates IL-4/IL-13 signaling and ameliorates experimentally induced allergic airway asthma in a murine model. Int Immunopharmacol, 2017; 50:305-12.

Ysebaert DK, De Greef KE, De Beuf A, Van Rompay AR, Vercauteren S, Persy VP, De Broe ME. T cells as mediators in renal ischemia/reperfusion injury. Kidney Int, 2004; 66(2):491-6; doi:10.1111/ j.1523-1755.2004.761_4.x
Zager RA, Johnson AC, Becker K. Renal cortical lactate dehydrogenase: a useful, accurate, quantitative marker of in vivo tubular injury and acute renal failure. PLoS One, 2013; 8(6):e66776.

Zeng W, Yan Y, Zhang F, Zhang C, Liang W. Chrysin promotes osteogenic differentiation via ERK/MAPK activation. Protein Cell, 2013; 4(7):539-47; doi:10.1007/s13238-013-3003-3.

Zhang H, Clemens RA, Liu F, Hu Y, Baba Y, Theodore P. STIM1 calcium sensor is required for activation of the phagocyte oxidase during inflammation and host defense. Blood, 2014; 123(14): 2238-49.

Zhang YL, Qiao SK, Wang RY, Guo XN. NGAL attenuates renal ischemia/reperfusion injury through autophagy activation and apoptosis inhibition in rats. Chem Biol Interact, 2018; 289:40-6.

How to cite this article:

Abdallah HMI, El Awdan SA, El-Marasy SA, Ahmed-Farid OA, Hassan A. Chrysin or mannitol for treatment of acute kidney injury: Evidence for pharmacokinetic interaction. J Appl Pharm Sci, 2021; 11(12):139-150. 